\title{
LOS PROBLEMAS DE LA VIOLENCIA Y LAS ADICCIONES EN EL CONTEXTO PERUANO
}

\section{VIOLENCE AND ADDICTION PROBLEMS IN THE PERUVIAN CONTEXT}

\author{
Fabián Fiestas ${ }^{1, a}$
}

Los tres pilares más importantes sobre los que la salud pública se sostiene, en mi opinión, son la ciencia, el altruismo y el activismo. La ciencia nos informa cuántos y quiénes son los que tienen un problema de interés, así como qué lo causa y bajo qué mecanismos para, finalmente, hacernos saber cuáles son las mejores medidas para controlarlo. De esta manera, por ejemplo, se ha generado el conocimiento necesario para la erradicación de la viruela, la reducción dramática de las muertes por tuberculosis o por el virus de la inmunodeficiencia humana, entre otros grandes logros. Pero la ciencia no puede sola, se necesita también del altruismo, entendido como la motivación y el deseo de ayudar que lleva a cada actor en salud a hacerse responsable del otro; y también del activismo, que logra que el conocimiento se llegue a traducir en acciones concretas. Entonces, son estos tres pilares los que este número de la RPMESP, dedicado a la violencia y las adicciones, trata de fortalecer y fomentar, ayudando así a que más acciones efectivas se empleen para controlar estos problemas de salud.

En el Perú, los trastornos por uso de sustancias psicoactivas y la violencia, son percibidas como prioridades sociales, aunque aún pareciera necesario reforzar la idea de que estas alteraciones pertenecen al área de la salud y, concretamente, al área de la salud mental. Quizá por ello esta percepción de prioridad todavía no termina de definirse en decisiones de acción y de presupuesto, lo cual hace que estos problemas sigan corriendo la misma suerte de olvido estructural tal como otras afecciones de salud mental. Y solo el estigma y la negación pueden explicar esta situación de olvido. De hecho, las alteraciones de salud mental explican el $16 \%$ de las pérdidas económicas por discapacidad y muerte prematura, lo que coloca a este grupo de enfermedades como la primera causa de carga de enfermedad en el Perú (1). No obstante ese costo, este grupo de problemas reciben menos del 2-3\% del presupuesto general de salud (2). Ello resulta aun más preocupante teniendo en cuenta que el Perú invierte poco en salud, como ejemplo, el 2009 se gastó solo el $4,6 \%$ del PBI, lo que lo coloca entre los cinco países de América que menos invierten en salud. Naturalmente, la consecuencia directa de este olvido estructural, es la persistencia y acentuación de problemas como la violencia y las adicciones.

Así, el olvido estructural en que se encuentra en general la salud mental explica el limitado acceso a tratamientos de calidad y a tiempo por parte de pacientes y familias, la falta de regulaciones legales y el reducido número de medidas de prevención implementadas basadas en la evidencia. Situaciones como las treinta muertes acontecidas por el incendio en una comunidad terapéutica establecida de manera informal en la capital peruana en enero de 2012, son el tipo de consecuencias que suceden cuando solo un fragmento muy pequeño de las personas que requieren un cuidado de salud mental, calculado para Perú en alrededor de $4 \%$, logran ser atendidos en algún servicio de salud ${ }^{(2)}$. Situaciones como la proliferación indiscriminada de centros de rehabilitación para adictos, u otros problemas mentales en todo el país ${ }^{(3)}$, son consecuencia de un sistema de salud pública que, como en el Perú, solo cuenta con 202 psiquiatras contratados para cubrir una población de más de 29 millones de personas, 175 de los cuales están concentrados en Lima, ciudad capital, mientras que once de los veintitrés departamentos restantes (incluyendo departamentos muy pobres y golpeados por serios problemas sociales, como Ayacucho, Ucayali y Madre de Dios) no tienen un solo psiquiatra contratado por el sistema público. A esto se añade el hecho de que la mayoría de médicos nopsiquiatras en el país no cuenta con capacitación suficiente como para afrontar problemas de salud mental.

\footnotetext{
1 Unidad de Análisis y Generación de Evidencias en Salud Pública, Centro Nacional de Salud Pública, Instituto Nacional de Salud. Lima, Perú.

a Médico epidemiólogo
} 
Los diferentes niveles en los que se manifiesta el olvido estructural en que se encuentran los problemas de salud mental como la adicción y la violencia, también incluye la falta de información disponible en cifras de monitoreo de estos problemas, producción de investigación y de revisiones sistemáticas para contar con la evidencia científica localmente contextualizada que informe la toma de decisiones. Aunque se han hecho varios estudios, especialmente de tipo epidemiológico, la producción ha sido poca y la información que se ha obtenido de la mayoría de ellos aún es muy limitada a cifras crudas como prevalencias o tablas descriptivas que, aunque importantes, no son suficientes para plantear soluciones puntuales, añadiéndose además el inconveniente que muchas veces la información aparece de forma dispersa $\mathrm{y}$, en algunos casos, duplicada y hasta inconsistente.

Esta situación tal vez explique por qué en el Perú se puedan dictaminar leyes tan separadas de lo mejor para la realidad local y de las buenas prácticas médicas internacionalmente aceptadas. Un ejemplo reciente de este tipo de regulaciones desconcertantes es la ley 29737, proclamada en julio de 2011 , donde se permite laxamente el internamiento involuntario de personas "con cualquier grado de adicción" que se puede autorizar únicamente con el consentimiento de familiares. Esta Ley, como varios otros ejemplos de políticas y medidas establecidas actualmente, solo puede ser explicada por un desconocimiento de la mejor evidencia científica respecto a tratamientos que sí son costo-efectivos. Por ejemplo, en este mismo número de la RPMESP, se presentan los resultados de una revisión sistemática de la eficacia de las comunidades terapéuticas (CT) para tratar problemas de consumo de sustancias, donde además de encontrar que no hay evidencia que las CT son superiores a otros tipos de tratamiento más sencillos, encuentra que ningún modelo de $\mathrm{CT}$ estudiado se considera el internamiento involuntario de los pacientes; incluso se presentan modelos ambulatorios y no residenciales. Estos resultados, que son consistentes con otras investigaciones ${ }^{(4)}$, no justifican científicamente un internamiento involuntario de pacientes con adicciones. Además, el internamiento involuntario es visto con mucha preocupación por la comunidad médica y grupos de la sociedad civil dado que puede significar una vulneración de los derechos básicos de los pacientes ${ }^{(5)}$.

Así, para evitar que se den y mantengan regulaciones y medidas fallidas, tiene que existir un contexto donde las instituciones técnicas y de abogacía sean sólidas, organizadas, y esgriman con la suficiente fuerza la evidencia científica como herramienta para informar las decisiones. Por ejemplo, es necesario que estas reconozcan y difundan que el actuar sobre estas áreas olvidadas de la salud significa también una inversión económica con alta posibilidad de disminuir costos para el Estado. Solo la violencia interpersonal le cuesta anualmente al Perú el $5,1 \%$ de su $\mathrm{PBI}{ }^{(6)}$, que es más de lo que se ha estado invirtiendo por año en todo el sector salud en las últimas décadas. $Y$, por el lado del consumo de sustancias psicoactivas, DEVIDA ha estimado que las pérdidas anuales causadas por este tipo de problemas alcanza al menos 444,7 millones de dólares americanos $(0,2 \%$ del $\mathrm{PBI})$, aunque quizá sea éste un cálculo subestimado, especialmente si tomamos en cuenta que solo los trastornos por uso de alcohol (que no incluyen los daños causados por el consumo de alcohol en ausencia de abuso o dependencia, como los accidentes de tránsito) significan la tercera causa de carga de enfermedad en el Perú. Entonces, actuar en estos problemas puede significar un gran ahorro a la economía de nuestra sociedad.

El presente número de la RPMESP nace del reconocimiento de la gran carga que significan la violencia y las adicciones a nuestra sociedad, y de la escasez de evidencia local que ayude a enfrentar estos problemas. Así, algunos puntos que resaltar de las investigaciones presentadas en este número están relacionados con la violencia contra la pareja, violencia infantil, uso de sustancias en jóvenes y tratamiento de adicciones presentando información que puede ser usada para enfocar acciones específicas desde la Salud Pública. Por ejemplo, los hallazgos de Blitchtein et al., y Fiestas et al., muestran con solidez que identificar y actuar sobre problemas como el consumo de alcohol ayudaría a prevenir casos de violencia contra la pareja; los motivos son varios: primero, la literatura científica concuerda con que el alcohol es factor causal de violencia, por lo que medidas a nivel macro que controlen el consumo de alcohol, como las presentadas en uno de los artículos de simposio en este número de la RPMESP, pueden tener un efecto positivo en reducir la frecuencia de violencia contra la pareja a nivel poblacional. Segundo, existen instrumentos confiables de tamizaje de consumo problemático de alcohol que son bastante sencillos de implementar y, tercero, contamos en el ámbito clínico con métodos de manejo para problemas por uso de alcohol efectivos y de bajo costo (como las intervenciones breves).

Uno de los aspectos más interesantes de este número, además, es que cuenta con dos artículos que usan bases de datos de estudios epidemiológicos realizados con presupuesto público, uno realizado por el Instituto Nacional de Estadística e Informática (INEI) y el otro auspiciado por el Ministerio del Interior. El que estas instituciones del Estado hayan hecho disponibles estas bases de datos a investigadores locales ha logrado que 
se produzca información que sin duda ayuda a la toma de decisiones y a ejercer una salud pública y políticas basadas en la evidencia. Así, sería una excelente iniciativa que también el Instituto Nacional de Salud Mental "Honorio Delgado-Hideyo Noguchi" o DEVIDA, hicieran públicas las bases de datos de los diferentes estudios epidemiológicos con información en salud mental realizados con dinero público, especialmente los realizados entre el 2002 y 2009, siguiendo así a la exitosa experiencia que tienen otros países, como EE. UU. y China, en producir evidencia local haciendo de libre e irrestricto el acceso a las bases de datos de estudios financiados con dinero de los contribuyentes.

También, en este número, el Lic. William Cabanillas, Psicólogo de la Comisión Nacional para el Desarrollo y Vida sin Drogas - DEVIDA, presenta un simposio donde muestra los aspectos que según su visión deben ser considerados cuando se trata de establecer estrategias para la prevención de drogas en el Perú. Asimismo, la Dra. Gloria Cueva, directora ejecutiva de la Dirección de Salud Mental del Ministerio de Salud del Perú (MINSA), presenta en el artículo de simposio "Violencia y adicciones: problemas de salud pública" las características, recursos y oportunidades en un marco conceptual para guiar las acciones a considerarse para enfrentar estos problemas en el país, e incluso señala ya algunos puntos que podrían estarse implementando en el corto plazo desde el MINSA.

Este número de la RPMESP, sin embargo, no cubre varios otros aspectos de la violencia de la que se necesita tener información para la toma de decisiones, como es el caso, por ejemplo, de la violencia de tipo delincuencial, la económica o la estructural. También se necesitan datos de epidemiología descriptiva en adicciones, como es el caracterizar subgrupos en mayor riesgo de tener problemas de uso de sustancias psicoactivas en población peruana. A pesar de los esfuerzos de difusión e invitación a participar en este número de la RPMESP, muy pocas instituciones abocadas a la salud mental en el Perú lo han hecho. Esperamos que estas instituciones se animen en el futuro a cubrir y publicar estudios en estas áreas, pues ello ayuda a hacer frente y controlar estos y otros problemas además de la violencia y las adicciones. En un país donde los recursos son escasos, y distribuidos inequitativamente como en el Perú, la evidencia científica localmente contextualizada es básica para aplicar medidas de salud pública de forma inteligente, que permita el máximo uso de esos escasos recursos. Pero, nuevamente, la ciencia no lo es todo. Nuestro altruismo y activismo será lo que haga que lo bueno para la sociedad, finalmente suceda.

\section{REFERENCIAS BIBLIOGRÁFICAS}

1. Velásquez A. La carga de enfermedad y lesiones en el Peru y las otras prioridades del plan esencial de aseguramiento universal. Rev Peru Med Exp Salud Publica. 2009;26(2):222-31.

2. Ministerio de Salud. Informe sobre los servicios de salud mental del subsector del Ministerio de Salud del Perú 2008. Lima: Ministerio de Salud del Perú; 2009.

3. Centro de Información y Educación para la Prevención del Abuso de Drogas (CEDRO). El problema de las drogas en el Perú. Lima: CEDRO; 2006.

4. Smith LA, Gates S, Foxcroft D. Therapeutic communities for substance related disorder. Cochrane Database Syst Rev. 2006;(1):CD005338.

5. Defensoría del Pueblo. Salud mental y derechos humanos: La situación de los derechos de las personas internadas en establecimientos de salud mental. Lima, Perú: Defensoría del Pueblo; 2005

6. Waters HR, Hyder AA, Rajkotia Y, Basu S, Butchart A. The costs of interpersonal violence--an international review. Health Policy. 2005;73(3):303-15.

\section{Consulte la versión electrónica de la Revista Peruana de Medicina Experimental y Salud Pública en} www.pubmed.gov

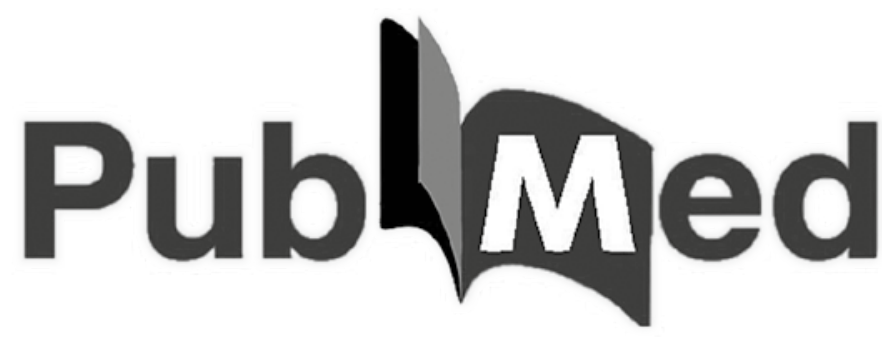

